\title{
МЕТОДИКА ЧИСЛЕННОГО ИССЛЕДОВАНИЯ ОТРАЖЕНИЯ УДАРНОЙ ВОЛНЫ ОТ ТВЕРДОЙ ПОВЕРХНОСТИ В ЗАПЫЛЁННОЙ СРЕДЕ
}

\author{
Д. А. Тукмаков ${ }^{\star}$, Н. А. Тукмакова ${ }^{\star *}$ \\ ${ }^{\star}$ Институт механики и машиностроения Казанского научного иентра \\ Российской академии наук \\ ${ }^{*}$ Казанский национальный исследовательский технический университет \\ (КНИТУ - КАИ) им. А. Н. Туполева
}

Поступила в редакцию 09.06.2019 г.

\begin{abstract}
Аннотация. В данной работе для исследования ударно-волновых течений в запылённых средах использована математическая модель динамики гетерогенной смеси. Гетерогенная смесь предполагалась состоящей из двух компонент: несущей компоненты - газа и дисперсной компоненты - твёрдых частиц. Для каждой из компонент среды решалась полная система уравнений динамики сплошной среды включавшая в себя уравнение неразрывности, уравнения сохранения пространственных компонент импульса, уравнение сохранения энергии. Несущая среда описывалась как вязкий, сжимаемый, теплопроводный газ. Математическая модель межкомпонентного силового взаимодействия включает в себя силу Стокса, динамическую силу Архимеда, силу присоединенных масс, а также теплообмен между компонентами смеси. Система уравнений решалась при помощи явного конечно-разностного метода Мак-Кормака второго порядка точности с применением схемы расщепления по пространственным направлениям. Для получения монотонного решения к сеточной функции применялась схема коррекции численного решения позволявшая уменьшить локальные максимумы и увеличить локальные минимумы искомых функций - преодолеть численную осцилляцию. С помощью программного кода реализующего численный алгоритм решения уравнений математической модели исследовалось влияние дисперсности частиц на параметры газа при отражении ударной волны от поверхности в запылённой среде. Ключевые слова: многофазные среды, явная конечно-разностная схема, коррекция численного решения, уравнение Навье-Стокса.
\end{abstract}

\section{ВВЕДЕНИЕ}

Многие явления и процессы, протекающие в естественной природе и технике, связаны с движением сплошных сред. При этом довольно часто исследуемые среды являются неоднородными по своим механическим и физико-химическим свойствам. В связи с этим одним из важных разделов современной механики жидкости и газа является динамика неоднородных сред. Экспериментальное исследование динамических процессов в неоднородных средах в ряде случаев затруднено, поэтому изучение динамики неоднородных сред требуют создания математических

( Тукмаков Д. А., Тукмакова Н. А., 2019 моделей [1-3]. Неоднородные среды могут быть смесью компонент, имеющих одинаковое агрегатное состояние - гомогенными смесями $[4,5]$ или же объединением компонент с разными агрегатными состояниями - гетерогенными смесями $[1-3,6-11]$.

Следует отметить, что математическое моделирование смесей с различным фазовым составом компонент является наиболее сложным в плане математического моделирования [8].

Особенностью динамики многокомпонентных смесей является необходимость учитывать эффекты, связанные с межкомпонентным взаимодействием. Учёт взаимодействия компонент смеси тем более важен, если различные компоненты имеют сопоставимые 
массовые доли, в таких смесях наблюдаются эффекты отличные от эффектов, выявленных в классической аэро и гидромеханике, так как динамика таких смесей во многом определяется взаимодействием компонент смеси. Примером таких смесей могут быть взвеси твёрдых или жидких дисперсных включений в газе - газовзвеси. Среды, состоящие из взвешенных в газе твёрдых частиц, называются запылёнными средами. В настоящее время в работах посвященных теоретическому исследованию динамики многофазных сред основной целью является разработка математических моделей течений гетерогенных смесей, как можно более полно учитывающих взаимодействие компонент смеси [6-11].

В литературе имеется несколько подходов к моделированию динамики неоднородных сред. В зависимости от параметров неоднородной среды это могут быть равновесный и неравновесный подходы. Равновесный подход предполагает исследование смеси как «квазиоднородной» то есть введение параметров учитывающих неоднородность среды и описание динамики такой системы как однородной жидкости или газа. Также имеется «континуальный» - неравновесный подход в котором описание движения каждой компоненты смеси требует решения полной системы уравнений динамики сплошной среды включающей в себя уравнения сохранения массы, импульса и энергии [2]. Подобный подход более предпочтителен для описания течений газовзвесей имеющих большое объёмное содержание дисперсной компоненты.

Интерес к математическому моделированию динамики газовзвесей вызван задачами, связанными с промышленными технологиями, оптимизацией установок транспорта дисперсных сред, оптимизацией технологий экранирования промышленных взрывов запылёнными средами, а также исследованием ударно-волновых течений многофазных сред в реактивных двигателях детонационного типа [6-11].

Математическая модель, применяемая в данной работе, позволяет учесть скоростную и тепловую неравновесность исследуемых процессов, межфазное силовое взаимодействие и межфазный теплообмен. Также несущая среда гетерогенной смеси описывается как вязкий сжимаемый и теплопроводный газ.

В данной статье изучается движение запылённой среды в ударной трубе- канале разделённом на две части, в одной части канала находился газ имеющий высокое давление, в другой части канала газ имеющий существенно меньшее давление и содержащий в себе взвешенные твёрдые частицы.

Как известно из классической газовой динамики, столкновение скачка уплотнения газа с твёрдой поверхностью приводит к формированию отраженного возмущения. Применяемая в исследовании математическая модель динамики газовзвеси учитывает взаимообратное влияние компонент на динамику гетерогенной смеси, что необходимо для исследования влияния свойств частиц дисперсной компоненты на параметры отраженной от твердой поверхности ударной волны, в запылённой среде, имеющей большое объёмное содержание дисперсной фазы.

\section{МЕТОДЫ ИССЛЕДОВАНИЯ}

В данной работе моделируется течение среды, представляющей собой газовую взвесь монодисперсного состава. Предполагается, что все включения дисперсной фазы имеют одинаковый размер и физические свойства материала, при этом со стороны несущей среды частицы находятся под действием силы аэродинамического сопротивления, силы Архимеда, силы присоединенных масс, также учитывается сила тяжести. Для описания движения двухфазной среды применяется система уравнений динамики многоскоростной и многотемпературной газовзвеси со скоростным и температурным скольжением фаз [1-3]. Математическая модель включает в себя уравнения движения несущей среды и дисперсной фазы. Одним из наиболее важных параметров дисперсной компоненты неоднородной смеси являлась «средняя плотность» - представляющая собой произведение объемного содержания дисперсной компоненты на физическую плотность материала дисперсной фазы $[1,2]$. Физическая 
Методика численного исследования отражения ударной волны от твердой поверхности ...

плотность материала дисперсных включений в процессе течения многофазной среды не изменяется. При этом объемное содержание является функцией временной и пространственных переменных.

Движение несущей среды описывается системой уравнений Навье-Стокса для сжимаемого теплопроводного газа с учетом межфазного силового взаимодействия и теплообмена $[12,13]$ :

$$
\begin{gathered}
\frac{\partial \rho_{1}}{\partial t}+\frac{\partial\left(\rho_{1} u_{1}\right)}{\partial x}+\frac{\partial\left(\rho_{1} v_{1}\right)}{\partial y}=0 \\
\frac{\partial\left(\rho_{1} u_{1}\right)}{\partial t}+\frac{\partial}{\partial x}\left(\rho_{1} u_{1}^{2}+p-\tau_{x x}\right)+ \\
+\frac{\partial}{\partial y}\left(\rho_{1} u_{1} v_{1}-\tau_{x y}\right)=-F_{x}+\alpha \frac{\partial p}{\partial x}, \\
\frac{\partial\left(\rho_{1} v_{1}\right)}{\partial t}+\frac{\partial}{\partial x}\left(\rho_{1} u_{1} v_{1}-\tau_{x y}\right)+ \\
+\frac{\partial}{\partial y}\left(\rho_{1} v_{1}^{2}-\tau_{y y}\right)=-\mathrm{F}_{y}+\alpha \frac{\partial p}{\partial y}, \\
\frac{\partial\left(e_{1}\right)}{\partial t}+\frac{\partial}{\partial x}\left(\left[e_{1}+p-\tau_{x x}\right] u_{1}-\tau_{x y} v_{1}+\lambda \frac{\partial T_{1}}{\partial x}\right)+ \\
+\frac{\partial}{\partial y}\left(\left[e_{1}+p-\tau_{y y}\right] v_{1}-\tau_{x y} u_{1}+\lambda \frac{\partial T_{1}}{\partial y}\right)= \\
=Q_{2}-\left|F_{x}\right|\left(u_{1}-u_{2}\right)-\left|F_{y}\right|\left(v_{1}-v_{2}\right)+ \\
+\alpha\left(\frac{\partial(p u)}{\partial x}+\frac{\partial(p v)}{\partial y}\right), \\
\tau_{x x}=\mu\left(2 \frac{\partial u_{1}}{\partial x}-\frac{2}{3} D\right), \tau_{y y}=\mu\left(2 \frac{\partial v_{1}}{\partial y}-\frac{2}{3} D\right) \\
=\mu\left(\frac{\partial u_{1}}{\partial y}+\frac{\partial v_{1}}{\partial x}\right), D=\frac{\partial u_{1}}{\partial x}+\frac{\partial v_{1}}{\partial y} .
\end{gathered}
$$

Динамика дисперсной фазы описывается уравнением сохранения средней плотности, уравнениями сохранения составляющих импульса и уравнением сохранения энергии, записанными с учетом теплообмена, обмена импульсом с несущей фазой:

$$
\begin{gathered}
\frac{\partial \rho_{2}}{\partial t}+\frac{\partial\left(\rho_{2} u_{2}\right)}{\partial x}+\frac{\partial\left(\rho_{2} v_{2}\right)}{\partial y}=0 \\
\frac{\partial\left(\rho_{2} u_{2}\right)}{\partial t}+\frac{\partial}{\partial x}\left(\rho_{2} u_{2}^{2}\right)+ \\
+\frac{\partial}{\partial y}\left(\rho_{2} u_{2} v_{2}\right)=F_{x}-\alpha \frac{\partial p}{\partial x}
\end{gathered}
$$

$$
\begin{gathered}
\frac{\partial\left(\rho_{2} v_{2}\right)}{\partial t}+\frac{\partial}{\partial x}\left(\rho_{2} u_{2} v_{2}\right)+ \\
+\frac{\partial}{\partial y}\left(\rho_{2} v_{2}^{2}\right)=F_{y}-\alpha \frac{\partial p}{\partial y}, \\
\frac{\partial\left(e_{2}\right)}{\partial t}+\frac{\partial}{\partial x}\left(e_{2} u_{2}\right)+\frac{\partial}{\partial y}\left(e_{2} v_{2}\right)=-Q_{2}, \\
\rho_{2}=\alpha_{2} \rho_{20}, e_{2}=\rho_{2} C_{v 2} T_{2},
\end{gathered}
$$

Силовое взаимодействие несущей среды и дисперсной компоненты описывается выражениями:

$$
\begin{gathered}
F_{x}=\frac{3 \alpha}{8 r} C_{d} \rho_{1} \sqrt{\left(u_{1}-u_{2}\right)^{2}+\left(v_{1}-v_{2}\right)^{2}}\left(u_{1}-u_{2}\right)+ \\
+\alpha \rho_{1}\left(\frac{\partial u_{1}}{\partial t}+u_{1} \frac{\partial u_{1}}{\partial x}+v_{1} \frac{\partial u_{1}}{\partial y}\right)+ \\
+0.5 \alpha \rho_{2}\left(\frac{\partial u_{1}}{\partial t}+u_{1} \frac{\partial u_{1}}{\partial x}+v_{1} \frac{\partial u_{1}}{\partial y}-\right. \\
\left.-\frac{\partial u_{2}}{\partial t}-u_{2} \frac{\partial u_{2}}{\partial x}-v_{2} \frac{\partial u_{2}}{\partial y}\right), \\
F_{y}=\frac{3 \alpha}{8 r} C_{d} \rho_{1} \sqrt{\left(u_{1}-u_{2}\right)^{2}+\left(v_{1}-v_{2}\right)^{2}}\left(v_{1}-v_{2}\right)+ \\
+\alpha \rho_{1}\left(\frac{\partial v_{1}}{\partial t}+u_{1} \frac{\partial v_{1}}{\partial x}+v_{1} \frac{\partial v_{1}}{\partial y}\right)- \\
+0.5 \alpha \rho_{1}\left(\frac{\partial v_{1}}{\partial t}+u_{1} \frac{\partial v_{1}}{\partial x}+v_{1} \frac{\partial v_{1}}{\partial y}-\right. \\
\left.-\frac{\partial v_{2}}{\partial t}-u_{2} \frac{\partial v_{2}}{\partial x}-v_{2} \frac{\partial v_{2}}{\partial y}\right)-\alpha \rho_{20} g, \\
V_{i}=\left[u_{i}, v_{i}\right], i=1,2 ; \mathrm{C}_{\mathrm{d} 2}=\frac{24}{\operatorname{Re}_{21}}+\frac{4}{\operatorname{Re}_{21}^{0.5}}+0.4, \\
M_{21}=\left|\bar{V}_{1}-\overline{V_{2}}\right| / c, \operatorname{Re}_{21}=\rho_{1}\left|\bar{V}_{1}-\overline{V_{2}}\right| 2 r / \mu, \\
\operatorname{Pr}=\gamma C_{p} \mu / \lambda .
\end{gathered}
$$

Здесь $p, \rho_{1}, u_{1}, v_{1}$ - давление, плотность, декартовы составляющие скорости несущей среды в направлении осей $x$ и $y$ соответственно; $T_{1}, e_{1}$-температура и полная энергия газа; $\rho_{2}, T_{2}, e_{2}, u_{2}, v_{2}$ - средняя плотность, температура, внутренняя энергия, декартовы составляющие скорости дисперсной фазы в направлении осей $x, y$. Температура несущей среды находится из формулы: $T_{1}=(\gamma-1)\left(e_{1} / \rho_{1}-0.5\left(u_{1}^{2}+v_{1}^{2}\right)\right) / R$, где $R-$ газовая постоянная несущей фазы. Внутренняя энергия взвешенной в газе дисперсной фазы определяется следующим образом - 


\section{Д. А. Тукмаков, Н. А. Тукмакова}

$e_{2}=\alpha \rho_{20} C_{p 2} T_{2}$, где $C_{p 2}$ - удельная теплоемкость единицы массы вещества дисперсной фазы. В уравнение энергии для несущей фазы входит коэффициент теплопроводности газа, коэффициент теплообмена $\alpha^{T}$ на поверхности частица - несущая среда и тепловой поток за счет теплообмена между газом и частицей $Q_{2}=\alpha^{T} 4 \pi r^{2}\left(T_{1}-T_{2}\right) n=6 \alpha \lambda N u\left(T_{1}-T_{2}\right) /(2 r)^{2}$, где $N u=2 r \alpha^{T} / \lambda$. Число Нуссельта определяется с помощью известной аппроксимации в зависимости от относительных чисел Маха, Рейнольдса и от числа Прандтля [2]:

$$
\begin{gathered}
N u=2 \exp \left(-M_{20}\right)+0.459 \operatorname{Re}_{20}^{0.55} \operatorname{Pr}^{0.33}, \\
0 \leq M_{21} \leq 2,0 \leq \operatorname{Re}_{21} \leq 2 \cdot 10^{5} .
\end{gathered}
$$

Система уравнений дополнялась соответствующими начальными и граничными условиями. На границах расчетной области задавались граничные условия Дирихле для составляющих скорости несущей и дисперсной фазы и граничные условия Неймана для остальных функций $[2,3]$.

Система уравнений (2)-(3) решалась с помощью явного конечно-разностного метода Мак-Кормака. Алгоритм явной схемы Мак-Кормака можно продемонстрировать на примере двумерного уравнения Бюргерса [12]:

$$
\begin{gathered}
\mathbf{q}_{t}+\mathbf{E}_{x}+\mathbf{F}_{y}=\mathbf{H} \\
\mathbf{q}=\left[\begin{array}{l}
u \\
v
\end{array}\right] ; \quad \mathbf{E}=\left[\begin{array}{l}
u v-v \partial u / \partial y \\
u^{2}-v \partial v / \partial y
\end{array}\right] \\
\mathbf{F}=\left[\begin{array}{l}
u\left(u^{2}+v^{2}\right) / 2 v \\
v\left(u^{2}+v^{2}\right) / 2 v
\end{array}\right]
\end{gathered}
$$

Явная схема Мак-Кормака для системы уравнений (4) включает в себя последовательно выполняемые шаги предиктор (5) и корректор (6) [8]:

$$
\begin{aligned}
\mathbf{q}_{\mathbf{j}, \mathbf{k}}^{*} & =\mathbf{q}_{\mathbf{j}, \mathbf{k}}^{\mathbf{n}}-\frac{\Delta t}{\Delta x}\left(\mathbf{E}_{\mathbf{j}+1, \mathbf{k}}^{\mathbf{n}}-\mathbf{E}_{\mathbf{j}, \mathbf{k}}^{\mathbf{n}}\right)- \\
& -\frac{\Delta t}{\Delta y}\left(\mathbf{F}_{\mathbf{j}, \mathbf{k}+\mathbf{1}}^{\mathbf{n}}-\mathbf{F}_{\mathbf{j}, \mathbf{k}}^{\mathbf{n}}\right)+\Delta t \mathbf{H}_{\mathbf{j}, \mathbf{k}}^{\mathbf{n}} \\
\mathbf{q}_{\mathbf{j}, \mathbf{k}}^{\mathbf{n}+\mathbf{1}}= & 0.5\left[\left(\mathbf{q}_{\mathbf{j}, \mathbf{k}}^{*}+\mathbf{q}_{\mathbf{j}, \mathbf{k}}^{\mathbf{n}}\right)-\frac{\Delta t}{\Delta x}\left(\mathbf{E}_{\mathbf{j}, \mathbf{k}}^{\mathbf{n}}-\mathbf{E}_{\mathbf{j}-1, \mathbf{k}}^{\mathbf{n}}\right)-\right. \\
- & \left.\frac{\Delta t}{\Delta y}\left(\mathbf{F}_{\mathbf{j}, \mathbf{k}}^{\mathbf{n}}-\mathbf{F}_{\mathbf{j}, \mathbf{k}-1}^{\mathbf{n}}\right)+\Delta t \mathbf{H}_{\mathbf{j}, \mathbf{k}}^{\mathbf{n}}\right]
\end{aligned}
$$

В расчетах применялась схема расщепления по пространственным переменным (7), реализуемая в виде симметричной последовательности одномерных операторов, выполняющих шаги предиктор и корректор, по соответствующим координатным осям, позволяющая построить решение на следующем временном слое [12-14]:

$\mathbf{q}_{j, k}^{n+1}=\mathbf{P}_{x}\left(\frac{\Delta t_{x}}{2}\right) \mathbf{P}_{y}\left(\frac{\Delta t_{y}}{2}\right) \mathbf{P}_{y}\left(\frac{\Delta t_{y}}{2}\right) \mathbf{P}_{x}\left(\frac{\Delta t_{x}}{2}\right) \mathbf{q}_{j, k}^{n}$

Переход со слоя $t^{n}$ на слой $t^{n+1}$ осуществляется следующим образом:

$$
\begin{aligned}
\mathbf{q}_{j, k}^{(1)} & =\mathbf{P}_{x}\left(\frac{\Delta t_{x}}{2}\right) \mathbf{q}_{j, k}^{n}, \mathbf{q}_{j, k}^{(2)}=\mathbf{P}_{y}\left(\frac{\Delta t_{y}}{2}\right) \mathbf{q}_{j, k}^{(1)}, \\
\mathbf{q}_{j, k}^{(3)} & =\mathbf{P}_{y}\left(\frac{\Delta t_{y}}{2}\right) \mathbf{q}_{j, k}^{(2)}, \mathbf{q}_{j, k}^{n+1}=\mathbf{P}_{x}\left(\frac{\Delta t_{x}}{2}\right) \mathbf{q}_{j, k}^{(3)} .
\end{aligned}
$$

Временные шаги $\Delta t_{x}=\Delta t_{y}=\Delta t$. Для получения вектора $\mathbf{q}^{(1)}$ нужно применить одномерный оператор $\mathbf{P}_{x}\left(\frac{\Delta t_{x}}{2}\right)$ по переменной $x$ к вектору газодинамических функций на временном слое $t^{n}$ и т. д.

Монотонность решения достигалась с помощью применения схемы коррекции [15] после перехода на новый временной слой при $t=t^{n+1}$. Алгоритм коррекции выполнялся последовательно вдоль координаты $x$, а затем вдоль координаты $y$ в расчетной области для функции $Z$ - одной из координат вектора $\mathbf{q}=\left(\rho_{1}, u_{1}, v_{1}, e_{1}, \rho_{2}, u_{2}, v_{2}, e_{2}\right)^{T}$. В случае если выполняются условия $\left(\delta Z_{j-1 / 2} \cdot \delta Z_{j+1 / 2}\right)<0$ или $\left(\delta Z_{j+1 / 2} \cdot \delta Z_{j+3 / 2}\right)<0$ то к функции $Z$ в $j$-м узле применяется алгоритм схемы коррекции:

$$
\tilde{Z}_{j}=Z_{j}+k\left(\delta Z_{j+1 / 2}-\delta Z_{j-1 / 2}\right) .
$$

Нижний индекс обозначает номер узла сетки соответственно вдоль $x$ или $y$.

Здесь использованы обозначения:

$$
\begin{gathered}
\delta Z_{j-1 / 2}=Z_{j}-Z_{j-1}, \\
\delta Z_{j+1 / 2}=Z_{j+1}-Z_{j}, \quad \delta Z_{j+3 / 2}=Z_{j+2}-Z_{j+1},
\end{gathered}
$$

$\tilde{Z}_{j}$ - значение функции после перехода на $(n+1)$-й временной слой по схеме Мак-Кормака, $k$ - коэффициент коррекции.

Численное решение проводилось на равномерной сетке с количеством узлов вдоль 
Методика численного исследования отражения ударной волны от твердой поверхности ...

оси $x-N_{x}=1000$ и количеством узлов вдоль оси $y-N_{y}=200$. Шаг по времени вычислялся исходя из условия Куранта - Фридрихса Леви [12]. Алгоритм численного решения системы уравнений математической модели был реализован на языке Fortran.

Численная модель неравновесной динамики газовзвеси основанная на явной схеме Мак-Кормака была тестирована сопоставлением результатов расчетов движения ударных волн по запылённым средам с известным из литературы численным решением полученным методом крупных частиц $[2,16]$.

Система уравнений дополнялась соответствующими начальными и граничными условиями. На границах расчетной области задавались граничные условия Дирихле для составляющих скорости несущей среды и фракций дисперсной фазы и граничные условия Неймана для остальных функций.

В статье [17] проведено сопоставление численных расчётов, полученных с помощью программного кода, реализующего численный алгоритм решения системы уравнений динамики монодисперсной газовзвеси с результатами физического эксперимента, по разлёту сжатого объёма газовзвеси в чистый газ [18], сопоставление показало приемлемое соответствие экспериментальных данных и численных расчётов. В экспериментальной работе были выявлены количественные и качественные отличия ударно-волновой динамики газовзвеси, от результатов расчётов движения чистого газа, что также выявлено в ряде публикаций в периодических изданиях [6-11].

\section{РЕЗУЛЬТАТЫ И ИХ ОБСУЖДЕНИЕ}

Моделировалось движение прямого скачка уплотнение из чистого газа в запылённую среду. Для давления газа и объёмного содержания дисперсной фазы задавались следующие соотношения в начальный момент времени:

$$
0 \leq x \leq 3.33, p(x, y)=588 \text { КПа, } \alpha=0 ;
$$

$$
3.33<x \leq 4, p(x, y)=98 \text { КПа, } \alpha=0.001 \text {. }
$$

Из рисунка и табл. 1 следует, что с уменьшением размера частиц монодисперсной га- зовзвеси интенсивность давления в отражённой ударной волне возрастает. При отражении ударной волны от твёрдой поверхности в мелкодисперсной газовзвеси, давление имеет большее значение, чем давление в отраженной ударной волне рассчитанное аналитически для чистого газа [19].

Таблица 1

Максимальное давление в отражённой ударной волне

\begin{tabular}{|l|l|}
\hline Чистый газ & $p=592$ КПа \\
\hline $\begin{array}{l}\text { Газовзвесь с диаметром } \\
\text { частиц 200 мкм }\end{array}$ & $p=610$ КПа \\
\hline $\begin{array}{l}\text { Аналитическое решение } \\
\text { для чистого газа }\end{array}$ & $p=611$ КПа \\
\hline $\begin{array}{l}\text { Газовзвесь с диаметром } \\
\text { частиц 20 мкм }\end{array}$ & $p=613 \mathrm{KПа}$ \\
\hline $\begin{array}{l}\text { Газовзвесь с диаметром } \\
\text { частиц 2 мкм }\end{array}$ & $p=625$ КПа \\
\hline
\end{tabular}

Уменьшение линейного размера твёрдых частиц в монодисперсной газовзвеси приводит к уменьшению скорости спутного потока, увеличению плотности и температуры газа в отраженной ударной волне - рис. 2 (а-в).

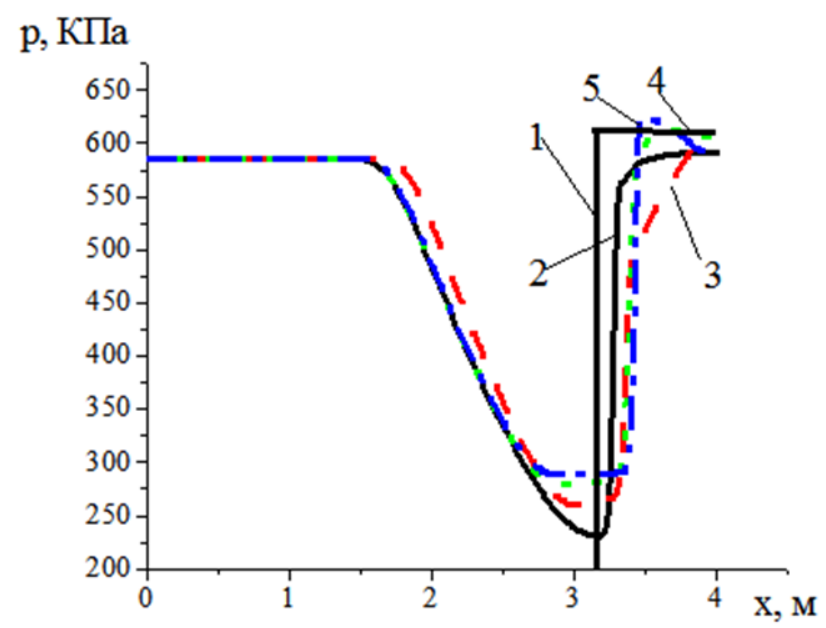

Рис. 1. Пространственное распределение давления в отражённой ударной волне кривая

1 - аналитическое решение, кривая

2 - численное решение для вязкого

однородного газа, кривая 3 - численное решение для газовзвеси с размером частии, 200 - мкм, кривая 4 - численное решение для газовзвеси с размером частии, 20 мкм, кривая

5 - численное решение для газовзвеси с размером частии 2 - мкм. 

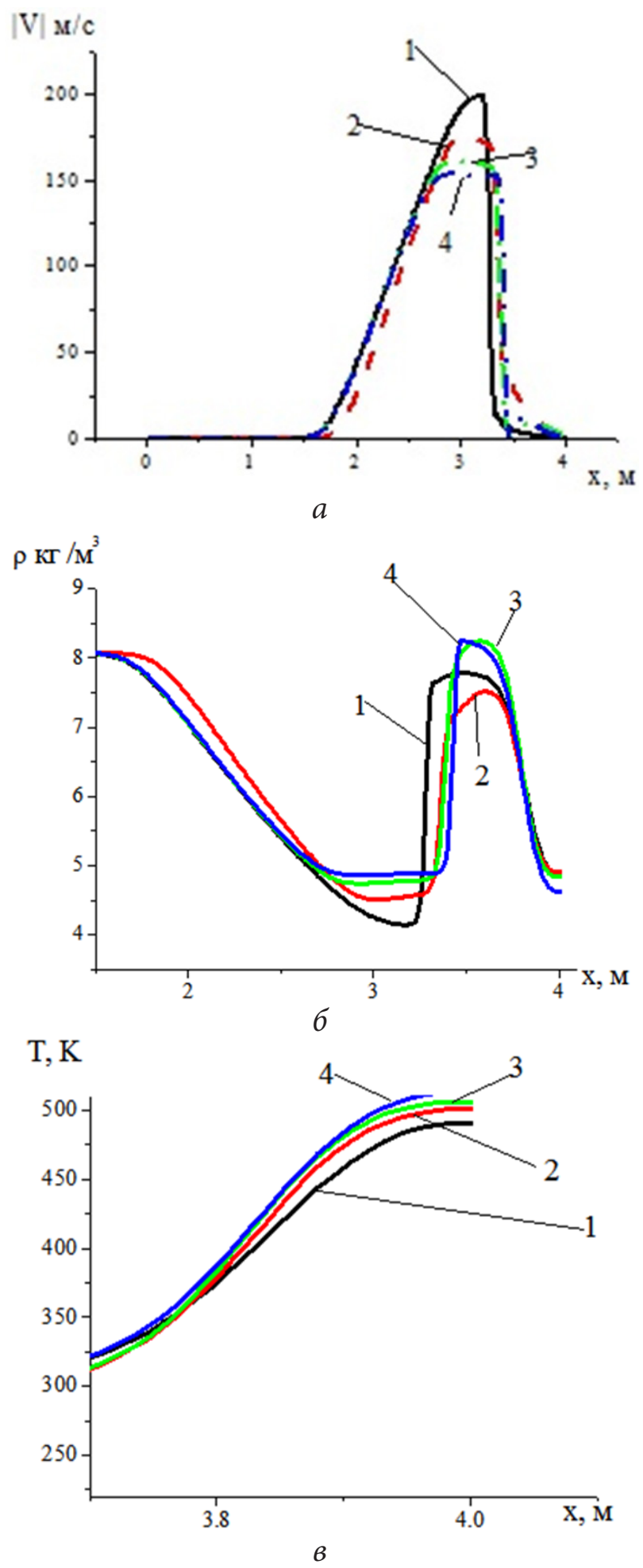

Рис. 2. Пространственное распределение модуля вектора скорости - рис. 3(a)

плотности - рис.3 (б) и температуры газа рис. 3 (в) в отражённой ударной волне; кривая

1 - численное решение для однородного газа, кривая 2-численное решение для газовзвеси

с размером частии, 200 - мкм, кривая 3 численное решение для газовзвеси с размером частии, 20 мкм

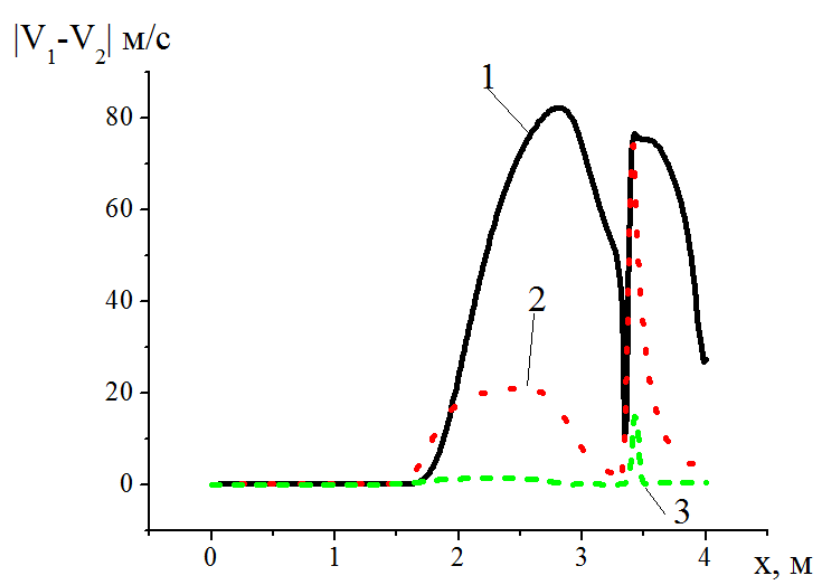

Рис. 3. Пространственное распределение модуля разности векторов скорости несущей и дисперсной базы в отражённой ударной волне кривая 1 - численное решение для газовзвеси с размером частии, 200 - мкм, кривая 2 - численное решение для газовзвеси с размером частии, 20 мкм, кривая 3 -численное решение для газовзвеси с размером частии, 2 мкм

Из рис. 3 следует, что для газовзвесей с более крупными частицами модуль разности векторов скоростей несущей и дисперсной фазы имеет большее значение.

Выявленные особенности отражения ударной волны в запылённых средах можно объяснить тем, что наличие дисперсной фазы приводит к замедлению течения газа, следствием чего является переход кинетической энергии несущей среды в потенциальную энергию, то есть рост температуры и давления газа. Замедление скорости движения газа в запылённой среде приводит к повышению плотности газа в волне сжатия, в сравнении со скоростью, в волне сжатия движущейся в чистом газе. В данной математической модели частицы имеют сферическую форму, то есть при одинаковом объёмном содержании уменьшение линейного размера частиц приводит к уменьшению площади соприкосновения одиночной частицы с газом в квадратичной пропорции и увеличению количества частиц в кубической пропорции. Таким образом, уменьшение размера частиц приводит к кратному увеличению площади соприкосновения несущей и дисперсной фазы, а, следовательно, к интенсификации межфазного взаимодействия и замедлению скорости движения газа. 
Методика численного исследования отражения ударной волны от твердой поверхности ...

\section{ВЫВОДЫ}

Анализ результатов численных расчётов показал, что уменьшение размера дисперсных частиц приводит к увеличению давления, температуры и плотности газа в отражённой ударной волне, уменьшению скорости движения отражённой ударной волны и уменьшению скорости спутного потока в отражённой волне. Выявленные закономерности отражения ударной волны в запылённой среде связаны с взаимообратным воздействием несущей и дисперсной компонент газовзвеси, что возможно выявить лишь с помощью применения математической модели гетерогенной смеси.

Работа выполнена при финансовой поддержке РФФИ Грант № 19-01-00442.

\section{СПИСОК ЛИТЕРАТУРЫ}

1. Нигматулин, Р. И. Основы механики гетерогенных сред: монография / Р. И. Нигматулин. - Москва : Наука, 1978. - 336 с.

2. Кутушев, А. Г. Математическое моделирование волновых процессов в аэродисперсных и порошкообразных средах: монография / А. Г. Кутушев. - Санкт-Петербург : Недра, 2003. - 284 с.

3. Федоров, А. В. Волновые процессы в газовзвесях частиц металлов: монография / А. В. Федоров, В. М. Фомин, Т. А. Хмель. - Новосибирск, 2015. - 301 с.

4. Павлов, В. А. Детонационное горение водорода в сопле Лаваля в условиях разряженной атмосферы / В. А. Павлов, Ю. В. Туник // Механика жидкости и газа. - 2012. № 5. - C. 99-106.

5. Применение детонации водородновоздушной смеси в устройствах для безыгольной инъекции / Голуб В. В. [и др.] // Теплофизика высоких температур. - 2013. - № 1. - С. 147150.

6. Веревкин, А. А. Течение дисперсной примеси в сопле Лаваля и рабочей секции двухфазной гиперзвуковой ударной трубы / А. А. Веревкин, Ю. М. Циркунов // Прикладная механика и техническая физика. - 2008. № 5. - С. 102-113.
7. Вараксин, Ю. А. Анализ механизмов осаждения твердых частиц на стенки каналов / Ю. А. Вараксин, М. В. Протасов, В. П. Яценко //Теплофизика высоких температур. - 2013. - № 5. -С. 738-746.

8. Садин, Д. В. TVD-схема для жестких задач волновой динамики гетерогенных сред негиперболического неконсервативного типа / Садин Д. В. // Журнал вычислительной математики и математической физики. - 2016. № 12. - С. 2098-2109.

9. Глазинов, А. А. Численное исследование течения ультрадисперсных частиц оксида алюминия в сопле ракетного двигателя твердого топлива / А. А. Глазинов, Н. Н. Дьяченко, Л. И. Дьяченко // Теплофизика и аэромеханика. - 2013. - № 1. - С. 81-88.

10. Арефьев, К. Ю. Расчетное исследование особенностей дробления и испарения капель в газодинамических течениях с циклическими ударными волнами / К. Ю. Арефьев, А. В. Воронецкий, С. А. Сучков // Известия высших учебных заведений. Машиностроение. - 2015. - № 10. - С. 17-30.

11. Hishida, $M$. Fundamentals of rotating detonations / M. Hishida, T.Fujiwara, P. Wolanski// Shock Waves. - 2009. - № 1. - P. 1-10.

12. Fletcher, C. A. Computation Techniques for Fluid Dynamics / C. A. Fletcher. - Berlin : Springer, 1988. - $502 \mathrm{p}$.

13. Ковеня, В. М. Применение метода расщепления в задачах аэродинамики / В. М. Ковеня, Г. А. Тарнавский, С. Г. Черный. - Новосибирск : Наука, 1990. - 247 с.

14. Тукмаков А. Л. Численное моделирование дрейфа твердых частиц при резонансных колебаниях газа в открытом канале / А. Л. Тукмаков // Акустический журнал. 2009. - № 2. - С. 247-255.

15. Музафаров, И. Ф. Применение компактных разностных схем к исследованию нестационарных течений сжимаемого газа / И. Ф. Музафаров, С. В. Утюжников// Математическое моделирование. - 1993. - № 3. C. 74-83.

16. Губайдуллин, Д. А. Численное исследование эволюции ударной волны в газовзвеси с учетом неравномерного распределения частиц / Д. А. Губайдуллин, Д. А. Тукмаков // 


\title{
Д. А. Тукмаков, Н. А. Тукмакова
}

Математическое моделирование. - 2014. № 10. - С. 109-119.

17. Нигматулин Р. И. Ударно-волновой раздет газовзвесей / Р. И. Нигматулин, Д. А. Губайдуллин, Д. А. Тукмаков //Доклады академии наук. - 2016. - № 4. - С. 418-421.

18. Ударные волны при разлете сжатого объема газовзвеси твёрдых частиц / Б. Е. Гель- фанд [и др.] // Доклады АН СССР. - 1985. № 5. - С. 1113-1116.

19. Овсянников, Л. В. Лекции по основам газовой динамики / Л. В. Овсянников. - Москва : Ижевск : Институт компьютерных исследований, 2003. - 336 с.

Тукмаков Д. А. - канд. физ.-мат. наук, н. с., Институт механики и машиностроения Федерального исследовательского центра «Казанский научный центр Российской академии наук». E-mail: tukmakovDA@imm.knc.ru

Тукмакова Н. А. - преподаватель, Казанский национальный исследовательский технический университет (КНИТУ - КАИ) им. А. Н. Туполева. E-mail: nadejdatukmakova@yandex.ru

\section{METHODOLOGY OF NUMERICAL RESEARCH OF REFLECTION OF A SHOCK WAVE FROM A SOLID SURFACE IN A DUSTY ENVIRONMENT}

\author{
D. A. Tukmakov*, N. A. Tukmakova ${ }^{\star *}$ \\ ${ }^{*}$ Institute of Mechanics and Engineering of Kazan Scientific Center of the Russian Academy of Sciences \\ ${ }^{*}$ Kazan National Research Technical University named after A.N. Tupolev - KAI
}

\begin{abstract}
Annotation. In this work, a mathematical model of the dynamics of a heterogeneous mixture was used to study shock-wave flows in dusty media. The heterogeneous mixture was assumed to consist of two components: the carrier component - gas and the dispersed component - solid particles. For each of the components of the medium, the complete system of equations of the continuum dynamics was solved, which included the continuity equation, the conservation equations for the spatial components of the momentum, and the energy conservation equation. The carrier medium was described as a viscous, compressible, heat-conducting gas. The mathematical model of the inter-component force interaction, which included the Stokes force, the dynamic force of Archimedes, the force of the added masses, as well as the heat exchange between the components of the mixture. The system of equations was solved using the Mac-Cormack explicit second-order finite-difference method using the splitting scheme in spatial directions. To obtain a monotonous solution to the grid function, a numerical solution correction scheme was applied, which allowed reducing local maxima and increasing local minima of the desired functions - overcoming numerical oscillation. Using the software code implementing the numerical algorithm for solving the equations of a mathematical model, the effect of particle dispersity on gas parameters during the reflection of a shock wave from a surface in a dusty medium was studied.
\end{abstract}

Keywords: multiphase media, explicit finite-difference scheme, numerical solution correction, Navier-Stokes equation.

Tukmakov D. A. - candidate of sciences, researcher - Institute of Mechanics and Engineering - a separate structural unit of the Federal State Budgetary Institution of Science "Federal Research Center" Kazan Scientific Center of the Russian Academy of Sciences"

E-mail: tukmakovDA@imm.knc.ru

Tukmakova N. A. - assistant, Kazan National Research Technical University named after A. N. Tupolev - KAI. E-mail: nadejdatukmakova@yandex.ru 